Article

\title{
Wide-Band Circularly Polarized ReflectarrayUsing Graphene-Based Pancharatnam-Berry Phase Unit-Cells for Terahertz Communication
}

\author{
Li Deng * (1), Yuanyuan Zhang, Jianfeng Zhu and Chen Zhang \\ Beijing Key Laboratory of Network System Architecture and Convergence, School of Information and \\ Communication Engineering, Beijing University of Posts and Telecommunications, Beijing 100876, China; \\ zhangyuanyuan@bupt.edu.cn (Y.Z.); zhujianfeng@bupt.edu.cn (J.Z.); zhangchenzc@bupt.edu.cn (C.Z.) \\ * Correspondence: dengl@bupt.edu.cn
}

Received: 2 May 2018; Accepted: 1 June 2018; Published: 5 June 2018

\begin{abstract}
A wide-band and high gain circularly polarized (CP) graphene-based reflectarray operating in the $\mathrm{THz}$ regime is proposed and theoretically investigated in this paper. The proposed reflectarray consists of a THz CP source and several graphene-based unit-cells. Taking advantages of the Pancharatnam Berry $(\mathrm{PB})$ phase principle, the graphene-based unit-cell is capable of realizing a tunable phase range of $360^{\circ}$ in a wide-band $(1.4-1.7 \mathrm{THz})$ by unit-cell rotating, overcoming the restriction of intrinsic narrow-band resonance in graphene. Therefore, this graphene-based unit-cell exhibits superior bandwidth and phase tunability to its previous counterparts. To demonstrate this, a wide-band (1.4-1.7 THz) focusing metasurface based on the proposed unit-cell that exhibits excellent focusing effect was designed. Then, according to the reversibility of the optical path, a CP reflectarray was realized by placing a wide-band $\mathrm{CP} \mathrm{THz} \mathrm{source} \mathrm{at} \mathrm{the} \mathrm{focal} \mathrm{point} \mathrm{of} \mathrm{the} \mathrm{metasurface.}$ Numerical simulation demonstrates that this reflectarray can achieve a stable high gain up to $15 \mathrm{dBic}$ and an axial ratio around $2.1 \mathrm{~dB}$ over the $1.4-1.7 \mathrm{THz}$ band. The good radiation performance of the proposed $\mathrm{CP}$ reflectarray, as demonstrated, underlines its suitability for the $\mathrm{THz}$ communication applications. Moreover, the design principle of this graphene-based reflectarray with a full $360^{\circ}$ phase range tunable unit-cells provides a new pathway to design high-performance $\mathrm{CP}$ reflectarray in the $\mathrm{THz}$ regime.
\end{abstract}

Keywords: circularly polarized; Pancharatnam Berry phase; graphene; metasurface

\section{Introduction}

Terahertz (THz) science and technology that is undergoing an unprecedented rapid development has attracted considerable attention in the scientific community. As a promising technology, it has enormous potential in applications such as communication [1], sensing [2], imaging [3], detection [4] and spectroscopy [5]. In terms of communication applications, the $\mathrm{THz}$ communication system is particularly promising as it has a sufficient bandwidth that is capable of handling high data rate up to $100 \mathrm{~Gb} / \mathrm{s}$ [6]. Consequently, new requirements for $\mathrm{THz}$ antennas have been put forward. Generally, high gain $\mathrm{THz}$ antennas are indispensable due to the intrinsic high path loss characteristic of $\mathrm{THz}$ waves in free space. Besides, considering the performance deterioration of linearly polarized antennas when they are polarization misaligned, circularly polarized $(\mathrm{CP})$ antennas are often required in scenarios where multi-path effect are prominent. Therefore, high gain $\mathrm{CP} \mathrm{THz}$ antennas are highly desirable.

Reflectarrays [7-12], which consist of an array of passive cells, are well suited for high gain $\mathrm{THz}$ applications $[13,14]$. They combine the merits of both conventional reflect and array antennas, namely, low loss, compact profile, low cross polarization, easy manufacturing, and high efficiency. 
By controlling the phase distribution on the array surface, the far-field radiation properties can be easily manipulated without bulky and lossy beamforming networks. In terms of CP reflectarray, several CP reflectarrays with excellent performances have been designed at microwave or millimeter wave frequencies [15-19]. It seems that reflectarrays can be chosen as a competitive candidate for high gain and CP THz applications. Nevertheless, most of these reflectarrays rely on judiciously designed metallic structures, which cannot simply scale to the $\mathrm{THz}$ frequencies because of the prominent skin effect of the conventional metal.The ohm loss of the metallic will immensely affect the array gain and radiation efficiency. Toavoid these drawbacks brought by the skin effect of metals at $\mathrm{THz}$ frequencies, there is a great demand for new types of $\mathrm{THz}$ antenna. Fortunately, graphene has several advantages over convention metals at the $\mathrm{THz}$ band. It has a high electron mobility up to 230,000 $\mathrm{cm}^{2} / \mathrm{Vs}$ at room temperature [20], and a low electrical resistivity about $10^{-6} \Omega \cdot \mathrm{cm}[21,22] \mathrm{in} \mathrm{THz}$ band, demonstrating lower loss than conventional metals. In addition, the surface plasmons resonant frequencies of graphene are quite lower than that in metals, which are often in optical frequencies. Meanwhile, graphene surface plasmons exhibit extremely small wavelengths $(\lambda / 10-\lambda / 100)$ and tight field confinement on the graphene sheet $[23,24]$, while maintaining reasonably small losses in the $\mathrm{THz}$ band. Furthermore, the imaginary conductivity of graphene is highly tunable via chemical doping or electrical gating [25-31] at THz frequencies, which is impossible or inefficient if metals are used. Based on excellent physical properties of graphene at THz frequencies, several graphene-based $\mathrm{THz}$ antennas have been reported in recent years [32-41]. It evaluates the feasibility of a fixed beam reflectarray antenna at $\mathrm{THz}$ based on graphene and compares its performance to a similar implementation using gold for the first time [42]. Soon after, diverse graphene-based reflectarrays operating at $\mathrm{THz}$ frequencies have been proposed [43-46]. As is well known, tunable unit-cell with a full $360^{\circ}$ reflected phase coverage is crucial for realizing a high-performance reflectarray. A small reflected phase tunable range of the unit-cell often leads to deteriorative radiation performance, limiting the function of the whole reflectarray. However, phase tunable ranges of unit-cells in previously reported graphene-based reflectarrays or even metasurfaces [47] are essentially realized by tuning physical parameters of the graphene-based structures. Thus, due to the intrinsic resonant properties and finite losses (such asa damped oscillator) of the graphene-based material, only a narrow band phase tunable range of $300^{\circ}$ has been presented [20,42-44], which restrict their applications in scenarios where high radiation performances are required. Especially, to the best of our knowledge, the CP graphene-based reflectarrays have also been seldom reported in previous literature, although there is a great demand on them at $\mathrm{THz}$ communications. One of the reason is that it is hard to achieve an excellent phase tunability by using graphene-based unit-cells. Therefore, designing a unit-cell with a wide-band tunable phase range of $360^{\circ}$ is a meaningful and challenging work for the graphene-based reflectarray design.

In this article, we propose a graphene-based wide-band $\mathrm{CP}$ reflectarray operating in the $\mathrm{THz}$ regime. The proposed reflectarray consists of a $\mathrm{THz} \mathrm{CP}$ source and several graphene-based unit-cells. Based on PB phase principle [48], the graphene-based unit-cell is designed to obtain a wide-band $(1.4-1.7 \mathrm{THz})$ tunable phase range of $360^{\circ}$, which overcomes the restriction of intrinsic narrow band resonant in graphene. Therefore, the graphene-based unit-cell exhibits superior bandwidth and phase tunability to its previous counterparts. Based on the proposed graphene-based unit-cell, a focusing metasurface operating in 1.4-1.7 THz bands is demonstrated firstly, which exhibits prominent focusing effect. Then, according to the reversibility of the optical path, we realize a CP reflectarray by placing a wide-band CP THz source at the focal point of the metasurface. Numerical simulation demonstrates that this reflectarray can achieve a stable high gain up to $15 \mathrm{dBic}$ and an axial ratio around 2.1 $\mathrm{dB}$ over the 1.4-1.7 THz bands. The good radiation performance of the proposed $\mathrm{CP}$ reflectarray, as demonstrated, underlines its suitability for the THz communication applications. 


\section{PB Principle}

To have a clear physical insight of the proposed CP reflectarray, firstly, we have deduced the principle of $\mathrm{PB}$ phase, obtaining the necessary phase requirement of the unit cell. As shown in Figure 1, we apply a rectangle to represent a PB unit cell. In Figure 1a, a right-handed CP (RHCP) incident wave normally (along the $-z$ direction) illuminates the surface, with an incident electric field expression

$$
E_{\mathrm{inc}}=(\vec{x}+j \vec{y}) e^{j \vec{k} \vec{z}}
$$

and the electric field of reflected wave will be

$$
E_{\mathrm{ref}}=\left(r_{x} \vec{x} e^{j \varphi_{x}}+j r_{y} \vec{y} e^{j \varphi_{y}}\right) e^{-j \vec{k} \vec{z}}
$$

where $\vec{k}$ denotes the wavenumber in free space. $r_{x}$ and $r_{y}$ represent the reflectivity of $x$-polarized and $y$-polarized waves, respectively. Similarly, $\varphi_{x}$ and $\varphi_{y}$ are the reflected phases of $x$-polarized and $y$-polarized waves, respectively.

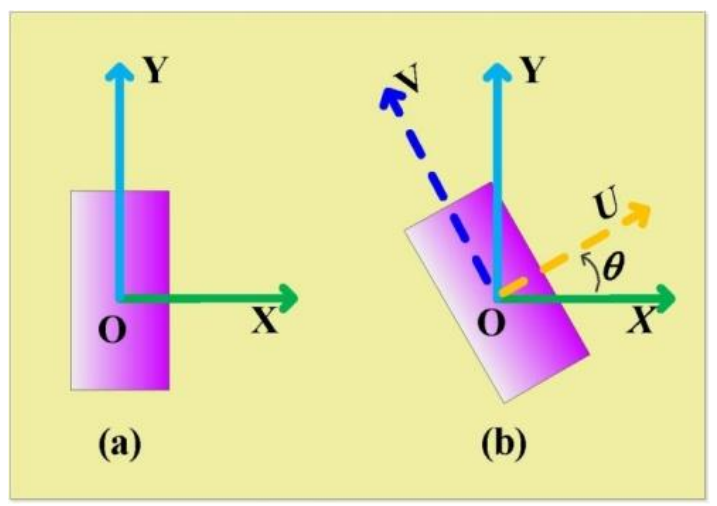

Figure 1. Illustration of $\mathrm{PB}$ phase unit-cell: (a) PB phase unit-cell in the $x y$ coordinate; (b) PB phase unit-cell in the $u v$ coordinate, which is transformed by an $\theta$ anticlockwise rotation of the original $x y$ coordinate. Both coordinates have the same original point.

As shown in Figure 1b, when the unit cell is anticlockwise rotated with an angle of $\theta$, the relationship between the $u v$ coordinate and the $x y$ coordinate can be represented by

$$
\left\{\begin{array}{l}
\vec{x}=\vec{u} \cos \theta-\vec{v} \sin \theta \\
\vec{y}=\vec{u} \sin \theta+\vec{v} \cos \theta
\end{array}\right.
$$

Thus, in the $u v z$ coordinate, we substitute Equation (3) into Equation (1), and the incident waves can be expressed as

$$
E_{\mathrm{inc}}=(\vec{u}+j \vec{v}) e^{j \vec{k}} \vec{z} e^{j \theta}
$$

and, corresponding reflected waves are

$$
E_{\mathrm{ref}}=\left(r_{u} \vec{u} e^{j \varphi_{u}}+j r_{v} \vec{v} e^{j \varphi_{v}}\right) e^{-j \vec{k} \vec{z}} e^{j \theta}
$$

From Figure 1, we can easily obtain that the reflectivity $r_{u}$ and reflected phase $\varphi_{u}$ in the $u v z$ coordinate are equal to the reflectivity $r_{x}$ and reflected phase $\varphi_{x}$ in the $x y z$ coordinate, respectively. In a similar way, the reflectivity $r_{v}$ and reflected phase $\varphi_{v}$ in the $u v z$ coordinate are equal to the 
reflectivity $r_{y}$ and reflected phase $\varphi_{y}$ in the $x y z$ coordinate, respectively. Therefore, $r_{u}=r_{x}, \varphi_{u}=\varphi_{x}$, $r_{v}=r_{y}$, and $\varphi_{v}=\varphi_{y}$. Then, Equation (5) can be expressed as

$$
E_{\mathrm{ref}}=\frac{1}{2}\left[\left(r_{x} \vec{x}-j r_{y} \vec{y}\right)\left(e^{j \varphi_{x}}-e^{j \varphi_{y}}\right) e^{j 2 \theta}+\left(r_{x} \vec{x}+j r_{y} \vec{y}\right)\left(e^{j \varphi_{x}}-e^{j \varphi_{y}}\right)\right] e^{-j \vec{k} \vec{z}}
$$

From Equation (6), if $r_{x}=r_{y}$, it is found that the reflected waves consist of two components, RHCP and left hand CP (LHCP), respectively.

$$
\begin{gathered}
E_{\mathrm{ref}(\mathrm{RHCP})}=\frac{1}{2} r_{x}(\vec{x}-j \vec{y})\left(e^{j \varphi_{x}}-e^{j \varphi_{y}}\right) e^{j 2 \theta} e^{-j \vec{k} \vec{z}} \\
E_{\mathrm{ref}(\mathrm{LHCP})}=\frac{1}{2} r_{x}(\vec{x}+j \vec{y})\left(e^{j \varphi_{x}}+e^{j \varphi_{y}}\right) e^{-j \vec{k} \vec{z}}
\end{gathered}
$$

and when $\left|\varphi_{x}-\varphi_{y}\right|=\pi$, Equations (7) and (8) change to

$$
\begin{aligned}
E_{\operatorname{ref}(\operatorname{RHCP})}= & \frac{1}{2} r_{x}(\vec{x}-j \vec{y}) e^{j \varphi_{x}} e^{-j \vec{k} \vec{z}} e^{j 2 \theta} \\
& E_{\mathrm{ref}(\mathrm{LHCP})}=0
\end{aligned}
$$

Obviously, there is only an RHCP component inside the reflected wave, and the phase variation is $2 \theta$, equal to two times of the rotation angle. Contrary to the metallic plate, the reflected waves of the $\mathrm{PB}$ unit-cell based metasurface only contain the co-polarized components. It is worth noting that the conditions of $\left|\varphi_{x}-\varphi_{y}\right| \approx \pi$ and $r_{x} \approx r_{y}$ must be fulfilled.

\section{Graphene Based PB Unit-Cell}

Graphene can strongly interact with electromagnetic waves in $\mathrm{THz}$ regime through plasmonic resonance $[23,24]$. However, for practical applications, wave-graphene interactions have to be further improved. In this paper, a graphene-based PB phase unit-cell is proposed, as shown in Figure $2 \mathrm{~b}$. The structure of the unit-cell consists of a top layer rectangular graphene patch and a square grounded quartz glass $\left(\mathrm{SiO}_{2}\right)$ substrate. When we extend these unit-cells periodically along both the $x$ and $y$ directions, a 2-D graphene-based metasurface can be obtained, as shown in Figure 2a. Incident terahertz waves can excite the plasmonic resonances of the graphene patches, and can be totally reflected by the bottom metallic ground. The top layer graphene-patches work as a partially reflecting mirror, and the bottom metallic ground operates as a fully reflecting mirror, respectively. Because of the rectangular shape of the graphene patch, the phases of the reflected waves can be independently manipulated by changing $w$ or $l$, as shown in Figure 2d. The reason is that the $E_{x}$ component of the incident wave can only excite the plasmonic resonance in the $x$ direction, and $E_{y}$ component of the incident wave can only excite the plasmonic resonance in the $y$ direction [49]. Based on this characteristic, we can easily design a unit-cell fulfilling the conditions of $\left|\varphi_{x}-\varphi_{y}\right| \approx \pi$ and $r_{x} \approx r_{y}$.

The modeling method of the graphene-based unit-cell is given in the following section. In the terahertz frequencies, the complex surface conductivity of graphene is determined by intraband transition. It can be approximated by a semi-classical Drude model [50]

$$
\sigma(\omega)=\frac{2 e^{2}}{\pi \hbar^{2}} k_{B} T \ln \left[2 \cosh \left(\frac{E_{f}}{2 k_{B} T}\right)\right] \frac{i}{\omega+i \tau^{-1}}
$$

where $e$ is the elementary charge, $k_{B}$ is the Boltzmann's constant, $\hbar$ is the reduced Plank's constant, $T$ is temperature, $\tau$ is the relaxation time, $\omega$ is the radian frequency, and $E_{f}$ is the Fermi energy. When $E_{f}$ is much larger than the thermal energy $k_{B} T$, the complex conductivity of graphene can be simplified to $\sigma(\omega)=\frac{e^{2} E_{f}}{\pi \hbar^{2}} \frac{i}{\omega+i \tau^{-1}}$. The electron relaxation time $\tau$ is the function of the carrier mobility $\mu$, the Fermi energy $E_{f}$, and Fermi velocity $v_{f}$. It can be expressed as $\tau=\frac{E_{f} \mu}{e v_{f}{ }^{2}}$, which implies that increasing 
$\mu$ will reduce the loss and enhance the efficiency of the device. The carrier mobility $\mu$ often has a variation range from $\sim 1000 \mathrm{~cm}^{2} / \mathrm{Vs}$ to $\sim 230,000 \mathrm{~cm}^{2} / \mathrm{Vs}$ with varied fabricated technologies [20]. In our simulation, according to the experimental results in Ref. [51], it is reasonable to assume that the Fermi energy $E_{f}=0.64 \mathrm{eV}$ (corresponding to electron concentration of $n=3 \times 10^{13} \mathrm{~cm}^{-2}$ in Ref. [51]), carrier mobility $\mu=10,000 \mathrm{~cm}^{2} / \mathrm{Vs}$, temperature $T=300 \mathrm{~K}$ and Fermi velocity $v_{f}=10^{6} \mathrm{~m} / \mathrm{s}$.
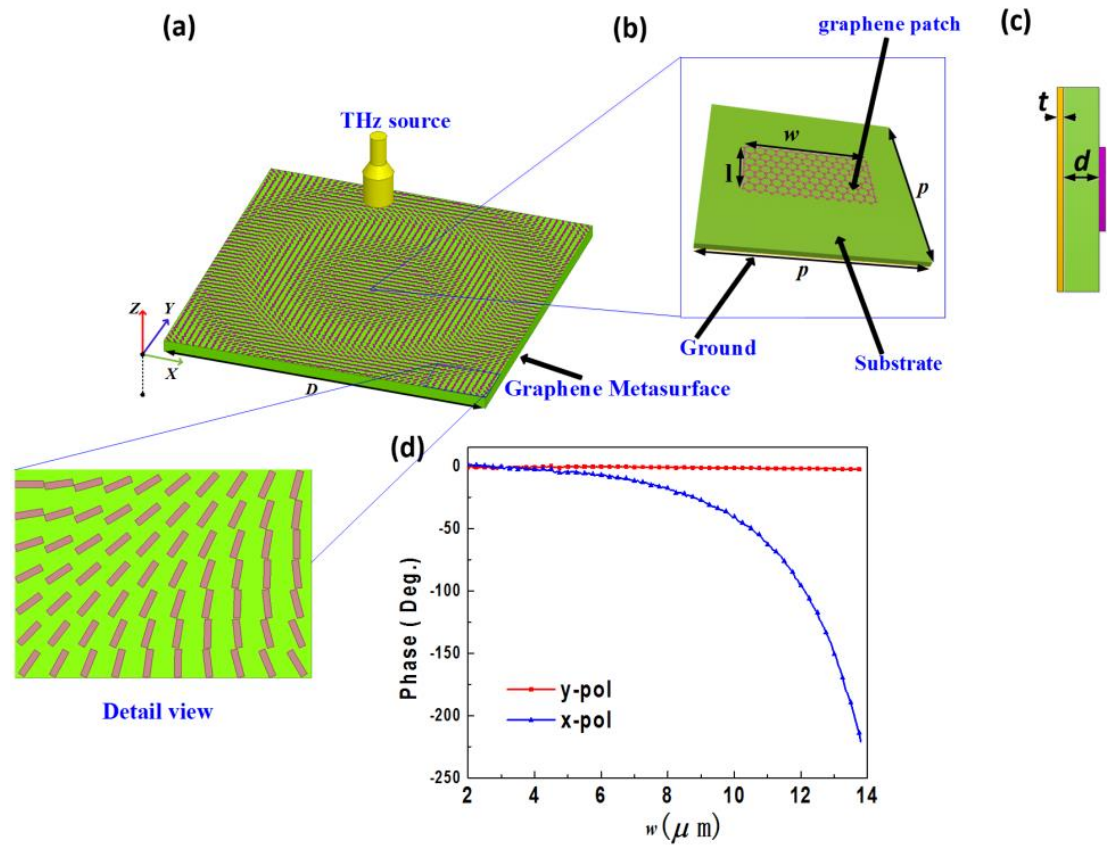

Figure 2. (a) Schematic of the proposed graphene-based reflectarray, which is composed of a focusing graphene metasurface and a CP THz source. (b) The PB unit-cell, which is composed of a rectangular graphene patch and a grounded quartz glass $\left(\mathrm{SiO}_{2}\right)$ substrate. The side lengths of the rectangle are $w$ and $l$, in $x$ and $y$ direction, respectively. $p$ represents the side length of the unit-cell, which can be extended in both $x$ and $y$ direction, forming a 2-D metasurface. (c) Side view of the proposed PB unit-cell. Geometric parameters $t$ and $d$ denote the thickness of the ground plate and substrate, respectively. (d) Simulated reflected phases under the normal illumination of the $x$-and $y$-polarized plane waves. Fixing $l=3.2 \mu \mathrm{m}$, the variation range of parameter $w$ is from $2 \mu \mathrm{m}$ to $13 \mu \mathrm{m}$, at $1.52 \mathrm{THz}$.

The reflectivity and phases of the proposed unit-cell were full-wave simulated using Ansoft HFSS 2017 software (Ansoft, Pittsburgh, PA, USA). The unit-cell depicted in Figure 2b,c is modeled with a graphene patch on the top layer, then it was deposited on a square grounded $\mathrm{SiO}_{2}$ substrate. The $\mathrm{SiO}_{2}$ substrate has a relative permittivity of $\varepsilon_{r}=3.75$, and a loss tangent $\tan \delta=0.0184$. The parameter $w$ and $l$ are the width and length of the graphene patch in the $x$ and $y$ directions, respectively, and $p=15 \mu \mathrm{m}$ denotes unit-cell side-length which also equal to a periodicity to form the 2-D metasurface. Besides, $t=10 \mathrm{~nm}$ and $d=26 \mu \mathrm{m}$ are the thickness of the metallic ground and quartz glass $\left(\mathrm{SiO}_{2}\right)$ substrate, respectively. In our simulation, the master and slaver boundary condition was added to the unit-cell to modeling an infinite array. Meanwhile, Floquet port is placed at $z=6 d$ and utilized to interact with the periodic unit-cell structure. In addition, the reflectivity and phases are obtained by the parametric sweep module of HFSS solver. The center frequency is set to $1.52 \mathrm{THz}$.

Such a PB unit cell can be looked as an anisotropic scatterer, which has a polarization dependent response. In normally incident case, we optimize the geometric parameters of the unit-cell, and choose the geometric parameters $w=13.39 \mu \mathrm{m}$, and $l=3.2 \mu \mathrm{m}$. Figure 3 a displays the reflectivity and reflected phases of the $x$ - and $y$-polarized waves, respectively. The reflectivity of $x$ - and $y$-polarized waves are almost equal, with values near 1 , indicating high efficiency. A nearly constant $180^{\circ}$ reflected phase 
difference between the $x$ - and $y$-polarized incident waves can be achieved in a wide frequency band $(1.4-1.7 \mathrm{THz})$, realizing exactly $180^{\circ}$ at $1.52 \mathrm{THz}$, as shown in Figure $3 \mathrm{~b}$.

According to the principle of PB phase, the proposed graphene-based unit-cell can realize co-polarized conversion, which can convert the illuminated $\mathrm{CP}$ wave into a co-polarized $\mathrm{CP}$ wave efficiently. Figure $3 c$ gives the co-polarized and cross-polarized conversion ratios when there is a normally RHCP incident wave, verifying the co-polarized conversion. In 1.4-1.7 THz bands, high-efficiency co-polarized transformation has been achieved. Parameters $r_{R R}$ and $r_{L R}$ indicate the co-polarized conversion ratio and cross-polarized conversion ratio, respectively.
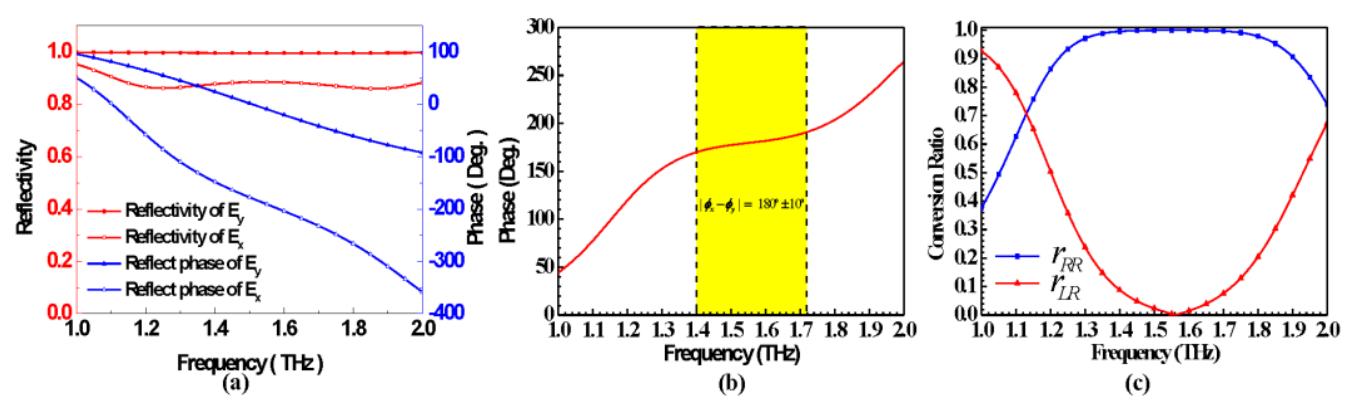

Figure 3. (a) Simulated reflection performance of the PB unit-cell under the normal illumination of $x$-and $y$-polarized plane waves. The red and blue curves represent the amplitude and phase of the reflected wave, respectively. Optimizing geometric parameters $w=13.39 \mu \mathrm{m}$ and $l=3.2 \mu \mathrm{m}$ are chosen in the simulation. (b) Simulated phase differences of the $x$-and $y$-polarized reflected waves. Yellow area in the figure demonstrates the frequency range of a nearly $180^{\circ}$ phase difference. (c) Simulated co-polarized and cross-polarized conversion ratios under the normal RHCP incident plane wave. The blue and red curves represent the co-polarized conversion ratio and cross-polarized conversion ratio, respectively.

\section{Graphene Metasurface for Focusing}

After verifying the wide-band high efficiency co-polarized converting capability of the proposed PB unit-cell, we can compensate arbitrary phase in a range of $0^{\circ}-360^{\circ}$ by element rotating.

$$
\varphi(x, y)=\frac{2 \pi}{\lambda_{0}}\left(\sqrt{x^{2}+y^{2}+f^{2}}-f\right)+\varphi_{0}
$$

To focus the reflected $\mathrm{CP}$ wave of a normally incident plane wave, a phase distribution must be fulfilled, where $f$ represents focal length, $\lambda_{0}$ represents wavelength in free space, $\varphi_{0}$ represents initial phase at the original point. Following Equation (12), we design a focusing graphene metasurface with $51 \times 51$ unit-cells at $1.52 \mathrm{THz}$, and the focal length is set to $190 \mu \mathrm{m}$. The phase distribution is illustrated in Figure 4a, and corresponding unit-cell distribution is plotted in Figure $4 \mathrm{~b}$. In the numerical simulation, a normally RHCP incident wave is placed along the $-z$ direction, obtaining energy distribution in the $x o z$ plane at 1.4, 1.52, 1.6 and $1.7 \mathrm{THz}$, respectively, as depicted in Figure 5a-d. From these results, the prominent focusing effect can be observed, verifying our design. Furthermore, as the frequency increases, the focal length increases proportional, in accord with Equation (12). It is worth noting that a full $360^{\circ}$ reflected phase range is quite important for generating such focusing metasurface. If narrower phase range is applied, as presented by previous studies [20,42,43], focusing performances will be degraded because of out of phase values in some positions of the metasurface. 


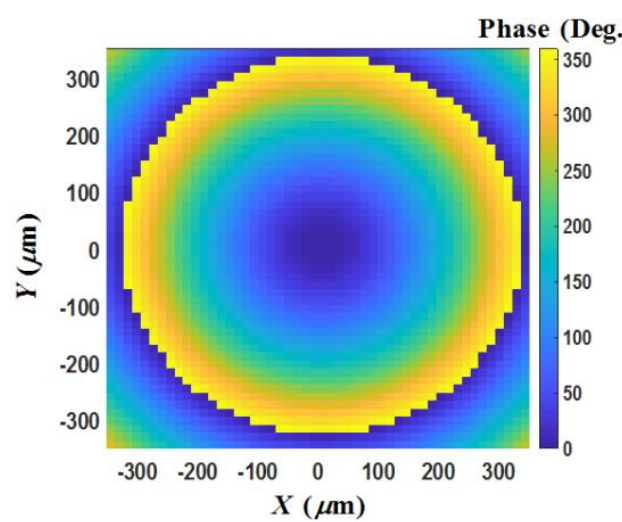

(a)

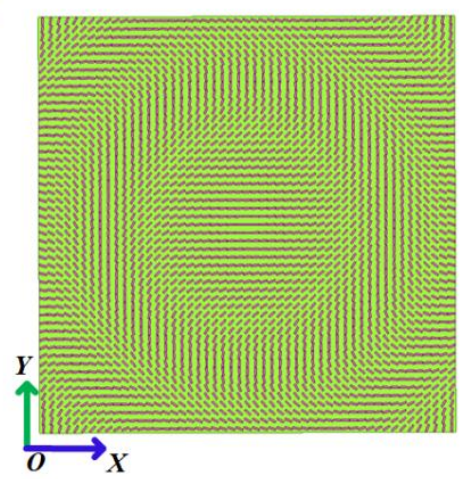

(b)

Figure 4. (a) The reflected phase distribution of the proposed focusing graphene metasurface with an area $714 \mu \mathrm{m} \times 714 \mu \mathrm{m}$. (b) The unit-cells distribution of the proposed focusing graphene metasurface with $51 \times 51$ unit-cells. Each unit-cell rotates a corresponding phase angle $\theta=\varphi / 2$.

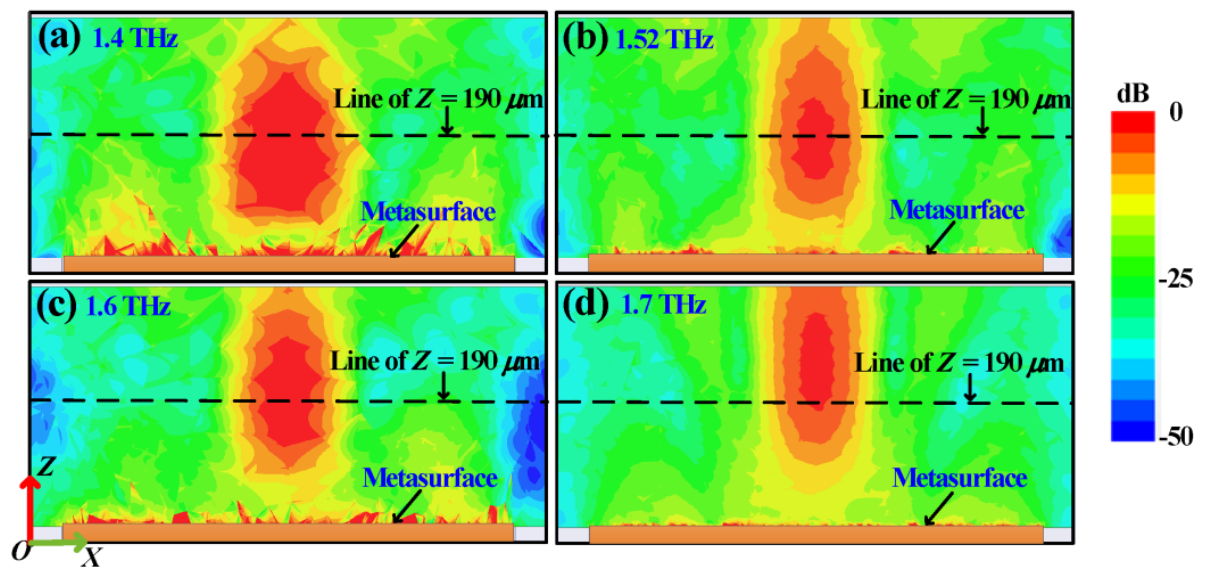

Figure 5. (a) Normalized energy distribution in $x o z$ plane at $1.4 \mathrm{THz}$, the center $z$ position of the focal point is less than $190 \mu \mathrm{m}$. (b) Normalized energy distribution in $x o z$ plane at $1.52 \mathrm{THz}$, the center $z$ position of the focal point is almost $190 \mu \mathrm{m}$. (c) Normalized energy distribution in xoz plane at $1.6 \mathrm{THz}$, the center $z$ position of the focal point is a little larger than $190 \mu \mathrm{m}$. (d) Normalized energy distribution in $x o z$ plane at $1.7 \mathrm{THz}$, the center $z$ position of the focal point is quite larger than $190 \mu \mathrm{m}$.

\section{High Gain CP Graphene-Based Reflectarray}

Previously, we have proposed a graphene metasurface which has prominent focusing capability in a wide-band of 1.4-1.7 THz. Then, according to the principle of optical path reversibility [52], we can place a wide-band CP THz source at the focal point of the metasurface. Thus, the incident spherical wave from the $\mathrm{THz}$ source can be converted to reflected plane wave with enhanced gain. In our simulation, we choose a $\mathrm{THz} \mathrm{CP}$ horn antenna as the feeding sourceand optimize its radiation pattern $(-10 \mathrm{~dB}$ beam width) for effective illumination. Simulated radiation patterns and axial ratios of the proposed feeding source at 1.4, 1.52, 1.6, and $1.7 \mathrm{THz}$ are demonstrated in Figures 6 and 7, respectively. Obviously, a stable $-10 \mathrm{~dB}$ RHCP beam-width around $120^{\circ}$ can be achieved, as shown in the figures. According to the calculated radiation angle, center feeding distance $190 \mu \mathrm{m}$ is chosen, in keeping with the focal length $f=190 \mu \mathrm{m}$ of the proposed metasurface. 

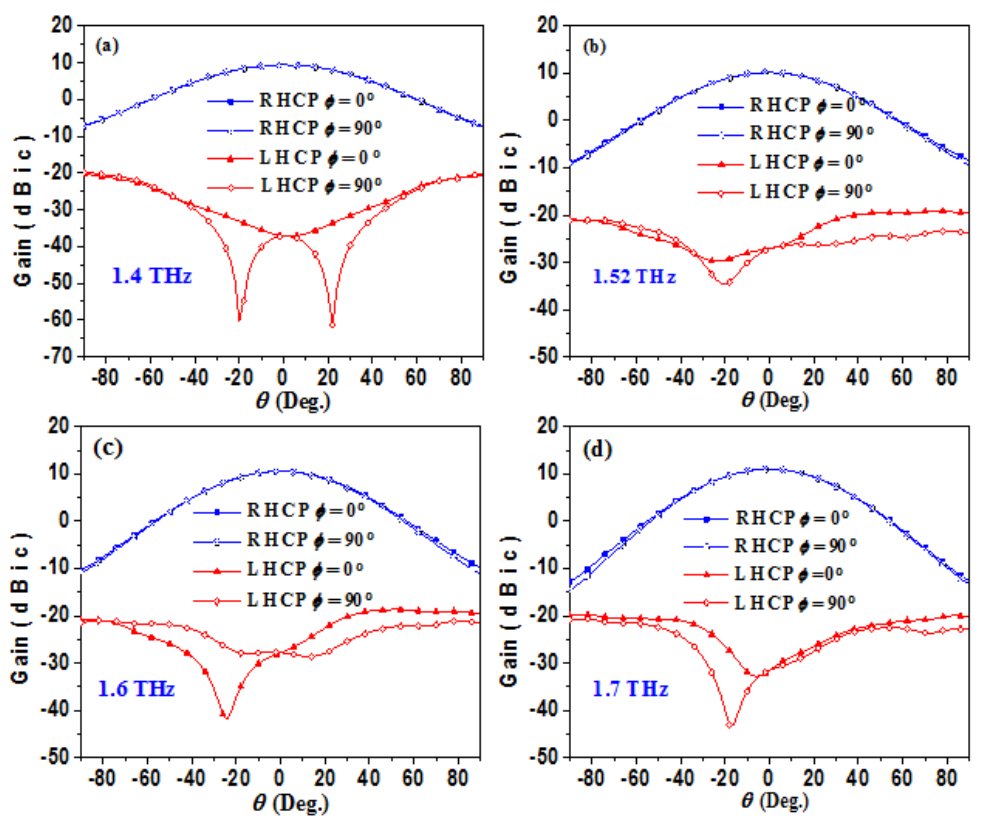

Figure 6. (a) Simulated 2-D radiation pattern of the feeding horn antenna at $1.4 \mathrm{THz}$. (b) The simulated 2-D radiation pattern of the feeding horn antenna at $1.52 \mathrm{THz}$. (c) The simulated 2-D radiation pattern of the feeding horn antenna at 1.6 THz. (d) The simulated 2-D radiation pattern of the feeding horn antenna at $1.7 \mathrm{THz}$. In all panels the gain of RHCP and LHCP components in $\varphi=0^{\circ}$ plane and $\varphi=90^{\circ}$ plane are demonstrated, respectively.
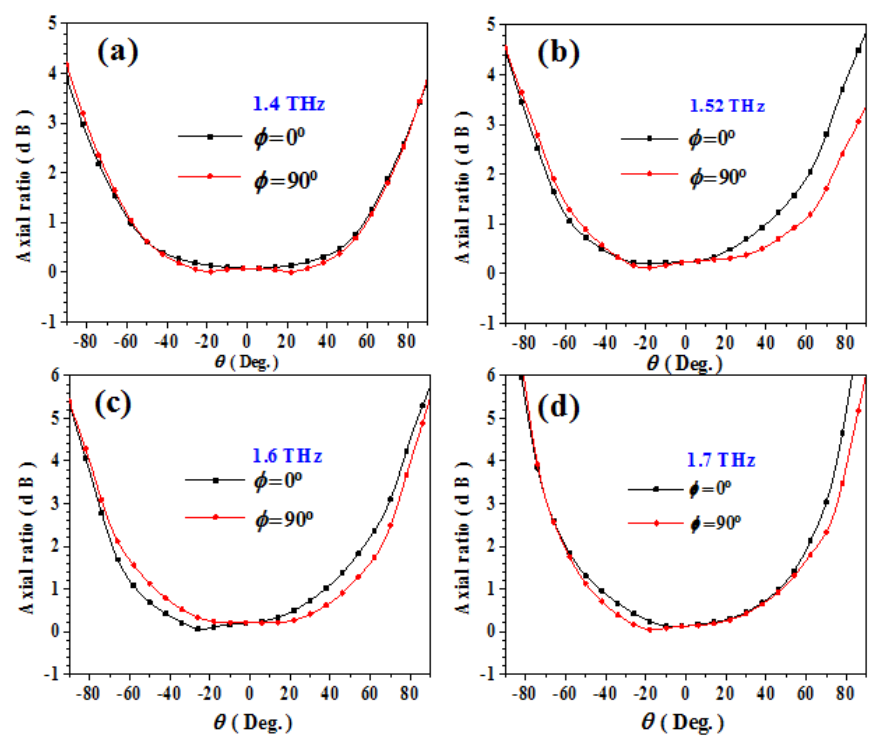

Figure 7. (a) Simulated axial ratio of the feeding horn antenna at $1.4 \mathrm{THz}$. (b) Simulated axial ratio of the feeding horn antenna at $1.52 \mathrm{THz}$. (c) Simulated axial ratio of the feeding horn antenna at $1.6 \mathrm{THz}$. (d) Simulated axial ratio of the feeding horn antenna at $1.7 \mathrm{THz}$. In all panels the axial ratios in $\varphi=0^{\circ}$ plane and $\varphi=90^{\circ}$ plane are demonstrated, respectively.

Figure $8 \mathrm{a}-1$ presents the radiation pattern of the reflectarray at $1.4,1.52,1.6$ and $1.7 \mathrm{THz}$, respectively. It is shown that the enhanced gains produced by the proposed reflectarray are 14.32, 15.34, 14.95 and $14.70 \mathrm{dBic}$, at 1.4, 1.52, 1.6 and $1.7 \mathrm{THz}$, respectively. Besides, the cross-polarization levels are at least $15 \mathrm{~dB}$ lower than the co-polarization levels all across the 1.4-1.7 THz bands, demonstrating good CP properties. It is worth noting that the co-polarization radiation patterns have little asymmetric 
feature. Due to the center-feeding regime $[53,54]$ of the proposed reflectarray, the CP feeding horn will inevitably interact with the reflected waves, causing distortion of the radiation pattern. Thus, we have to optimize the design parameters to maximally reduce the blockage effect of the feeding structure. Besides, we can alleviate such interaction by off-axial feeding regime. Furthermore, wide-band gain and axial ratio results are displayed in Figure 9, stable gain around $15 \mathrm{dBic}$ and axial ratio around $2.1 \mathrm{~dB}$ can be achieved in the whole 1.4-1.7 THz bands, exhibiting both high gain and $\mathrm{CP}$ capability, simultaneously.
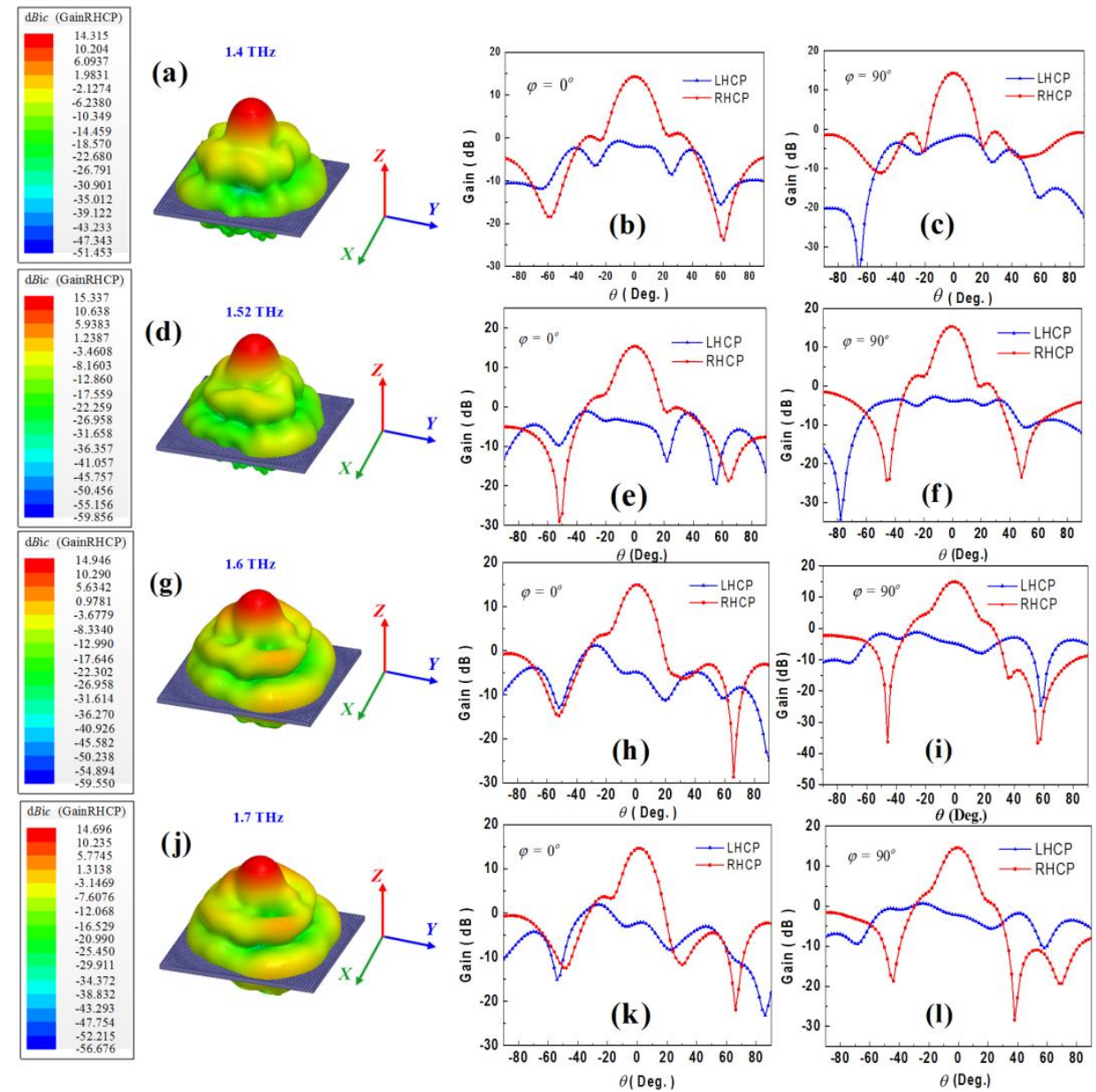

Figure 8. (a) 3-D radiation pattern of the proposed reflectarray at $1.4 \mathrm{THz}$. (b) 2-D radiation pattern of the proposed reflectarray at $1.4 \mathrm{THz}$ in $\phi=0^{\circ}$ plane. (c) 2-D radiation pattern of the proposed reflectarray at $1.4 \mathrm{THz}$ in $\phi=90^{\circ}$ plane. (d) 3-D radiation pattern of the proposed reflectarray at $1.52 \mathrm{THz}$. (e) 2-D radiation pattern of the proposed reflectarray at $1.52 \mathrm{THz}$ in $\phi=0^{\circ}$ plane. (f) $2-\mathrm{D}$ radiation pattern of the proposed reflectarray at $1.52 \mathrm{THz}$ in $\phi=90^{\circ}$ plane. (g) 3-D radiation pattern of the proposed reflectarray at $1.6 \mathrm{THz}$. (h) 2-D radiation pattern of the proposed reflectarray at $1.6 \mathrm{THz}$ in $\phi=0^{\circ}$ plane. (i) 2-D radiation pattern of the proposed reflectarray at $1.6 \mathrm{THz}$ in $\phi=90^{\circ}$ plane. (j) 3-D radiation pattern of the proposed reflectarray at $1.7 \mathrm{THz}$. (k) 2-D radiation pattern of the proposed reflectarray at $1.7 \mathrm{THz}$ in $\phi=0^{\circ}$ plane. (1) 2-D radiation pattern of the proposed reflectarray at $1.7 \mathrm{THz}$ in $\phi=90^{\circ}$ plane. 


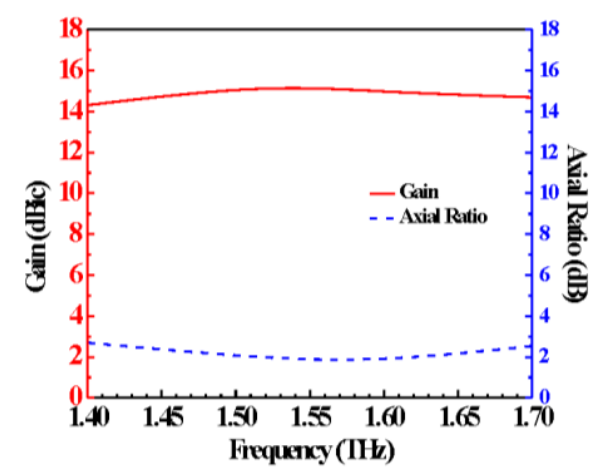

Figure 9. Simulated RHCP gain and axial ratio of the proposed reflectarray. The red and the blue curves represent the RHCP gain and axial ratio, respectively.

\section{Conclusions}

We have systematically investigated the spectral responses of graphene-based PB phase unit-cell and demonstrated a practical implementation of wide-band high gain $\mathrm{CP}$ reflectarray based on this configuration in the $\mathrm{THz}$ regime. Based on $\mathrm{PB}$ phase principle, an arbitrary reflected phase controlling can be realized, extremely extending the phase modulated ability of conventional graphene-based unit-cells that only have a phase controlling range of $300^{\circ}$. Finally, a focusing graphene-based metasurface and a reflectarray were designed, simulated and optimized. Simulation results exhibit excellent performances as theoretical expectations. The proposed reflectarray has a stable gain of $15 \mathrm{dBic}$ and axial ratio of $2 \mathrm{~dB}$ over the 1.4-1.7 THz bands, demonstrating high gain and $\mathrm{CP}$ characteristics, simultaneously. This reflectarray may have a promising application in future $\mathrm{THz}$ communications. Meanwhile, for practical fabrication, we can design the graphene-based unit-cell which is consist of five layers, such as graphene layer, alumina layer, polysilicon layer, quartz glass layer, and ground. The polysilicon can be applied as an electrode. The Fermi energy is related to the conductivity of graphene, and can be dynamically tuned by varying the DC voltage (VDC) between the graphene and the polysilicon. Detailed techniques to fabricate these kinds of graphene reflectarray can be found in Reference [55], supporting the feasibility of our design. In addition, it is worth noting that the general technical procedure formulated herein facilitates further production of such graphene-based devices for various applications.

Author Contributions: L.D. proposed the idea and designed the structure; Y.Z. and J.Z. analyzed the data; and L.D. and C.Z. wrote the paper.

Funding: This research was funded by National Natural Science Foundation of China (No. 61601040).

Conflicts of Interest: The authors declare no conflict of interest.

\section{References}

1. Petrov, V.; Komarov, M.; Moltchanov, D.; Jornet, J.M.; Koucheryavy, Y. Interference and SINR in millimeter waveand terahertz communication systems with blocking and directional antennas. IEEE Trans. Wirel. Commun. 2017, 16, 1791-1808. [CrossRef]

2. Singh, L.; Xie, L.; Chen, M.; Xu, N.; Singh, R.; Zhang, W. Terahertz sensing of highly absorptive water-methanol mixtures with multiple resonances in metamaterials. Opt. Express 2017, 25, 14089-14097.

3. Suen, J.Y.; Fan, K.; Padilla, W.J.; Liu, X. All-dielectric metasurface absorbers for uncooled terahertz imaging. Optica 2017, 4, 601.

4. Murano, K.I.; Watanabe, A.; Kasamatsu, S.; Suzuki, M. Low-profile terahertz radar based on broadband leaky-wave beam steering. IEEE Trans. Terahertz Sci. Technol. 2017, 7, 60-69. [CrossRef]

5. Chopra, N.; Yang, K.; Abbasi, Q.H.; Qaraqe, K.A.; Philpott, M.; Alomainy, A. THz time-domain spectroscopy ofhuman skin tissue for in-body nanonetworks. IEEE Trans. Terahertz Sci. Technol. 2016, 6, 803-809. [CrossRef] 
6. Koenig, S.; Lopezdiaz, D.; Antes, J.; Boes, F.; Henneberger, R.; Leuther, A. Wireless sub-THZ communication system with high data rate. Nat. Photonics 2013, 7, 977-981. [CrossRef]

7. Hu, W.; Cahill, R.; Encinar, J.A. Design and measurement of reconfigurable millimeter wave reflectarray cells with nematic liquid crystal. IEEE Trans. Antennas Propag. 2008, 56, 3112-3117. [CrossRef]

8. Headland, D.; Carrasco, E.; Nirantar, S.; Withayachumnankul, W.; Gutruf, P.; Schwarz, J.; Abbott, D.; Bhaskaran, M.; Sriram, S.; Perruisseau-Carrier, J.; et al. Dielectric resonator reflectarray as high-efficiency nonuniform terahertz metasurface. ACS Photonics 2016, 3, 1019-1026. [CrossRef]

9. Alizadeh, P.; Parini, C.G.; Rajab, K.Z. Optically reconfigurable unit cell for Ka-band reflectarray antennas. Electron. Lett. 2017, 53, 1526-1528. [CrossRef]

10. Encinar, J.A.; Datashvili, L.S.; Zornoza, J.A. Dual-polarization dual-coverage reflectarray for space applications. IEEE Trans. Antennas Propag. 2006, 54, 2827-2837. [CrossRef]

11. Florencio, R.; Encinar, J.A.; Boix, R.; Losada, R.V. Reflectarray antennas for dual polarization and broadband telecom satellite applications. IEEE Trans. Antennas Propag. 2015, 63, 1234-1246. [CrossRef]

12. Chou, H.T.; Ho, H.K.; Chen, Y.J. Radiation discrepancy analysis for metallic reflectarray antennas with random manufacture distortion at mmW frequencies. IEEE Antennas Wirel. Propag. Lett. 2016, 15, 1885-1888. [CrossRef]

13. Chen, L.; Qu, S.W.; Chen, B.J. Terahertz metasurfaces for absorber or reflectarray applications. IEEE Trans. Antennas Propag. 2017, 65, 234-241. [CrossRef]

14. Hasani, H.; Tamagnone, M.; Capdevila, S. Tri-band, polarization-independent reflectarray at terahertz frequencies: Design, fabrication, and measurement. IEEE Trans. Terahertz Sci. Technol. 2016, 6, 268-277. [CrossRef]

15. Han, C.; Rodenbeck, C.; Huang, J. A C/Ka dual frequency dual layer circularly polarized reflectarray antenna with microstrip ring elements. IEEE Trans. Antennas Propag. 2004, 52, 2871-2876. [CrossRef]

16. Mener, S.; Gillard, R.; Sauleau, R. Dual circularly polarized reflectarray with independent control of polarizations. IEEE Trans. Antennas Propag. 2015, 63, 1877-1881. [CrossRef]

17. Deng, R.; Mao, Y. A single-layer dual-band circularly polarized reflectarray with high aperture efficiency. IEEE Trans. Antennas Propag. 2015, 63, 3317-3320. [CrossRef]

18. Abadi, S.M.; Amin, M.H.; Behdad, N. Broadband true-time-delay circularly polarized reflectarray with linearly polarized feed. IEEE Trans. Antennas Propag. 2016, 64, 4891-4896. [CrossRef]

19. Haraz, O.M. A millimeter-wave circular reflectarray antenna for future 5G cellular networks. In Proceedings of the 2015 IEEE International Symposium on Antennas and Propagation \& USNC/URSI National Radio Science Meeting, Vancouver, BC, Canada, 19-24 July 2015; pp. 1534-1535.

20. Li, Z.; Yao, K.; Xia, F.; Shen, S.; Tian, J.; Liu, Y. Graphene plasmonic metasurfaces to steer infrared light. Nano Lett. 2015, 5, 12423. [CrossRef] [PubMed]

21. Jablan, M.; Buljan, H. Plasmonics in graphene at infrared frequencies. Phys. Rev. B 2009, 80, $196-206$. [CrossRef]

22. Koppens, F.H.; Chang, D.E.; García de Abajo, F.J. Graphene plasmonics: A platform for strong light-matter interactions. Nano Lett. 2011, 11, 3370-3377. [CrossRef] [PubMed]

23. Fei, Z.; Rodin, A.S.; Andreev, G.O.; Bao, W.; McLeod, A.S.; Wagner, M.; Zhang, L.M.; Zhao, Z.; Thiemens, M.; Dominguez, G.; et al. Gate-tuning of graphene plasmons revealed by infrared nano-imaging. Nature 2012, 487, 82-85. [CrossRef] [PubMed]

24. Chen, J.; Badioli, M.; AlonsogonzÃalez, P.; Thongrattanasiri, S.F. Optical nano-imaging of gate-tunable graphene plasmons. Nature 2012, 487, 77-81. [CrossRef] [PubMed]

25. Liu, P.Q.; Luxmoore, I.J.; Mikhailov, S.A. Highly tunable hybrid metamaterials employing split-ring resonators strongly coupled to graphene surface plasmons. Nat. Commun. 2015, 6, 8969. [CrossRef] [PubMed]

26. Wang, F.; Zhang, Y.; Tian, C. Gate-variable optical transitions in graphene. Science 2008, 320, $206-209$. [CrossRef] [PubMed]

27. Youngblood, N.; Anugrah, Y.; Ma, R. Multifunctional graphene optical modulator and photodetector integrated on silicon waveguides. Nano Lett. 2014, 14, 2741-2746. [CrossRef] [PubMed]

28. Peres, N.M.; Ribeiro, R.M.; Castro, N.A.H. Excitonic effects in the optical conductivity of gated graphene. Phys. Rev. Lett. 2010, 105, 055501. [CrossRef] [PubMed] 
29. Giovannetti, G.; Khomyakov, P.A.; Brocks, G. Doping graphene with metal contacts. Phys. Rev. Lett. 2008, 101, 026803. [CrossRef] [PubMed]

30. Zhang, Y.; Tang, T.T.; Girit, C.Z. Direct observation of a widely tunable bandgap in bilayer graphene. Nature 2009, 459, 820-823. [CrossRef] [PubMed]

31. Avouris, P.; Freitag, M. Graphene photonics, plasmonics, and optoelectronics. IEEE J. Sel. Top. Quantum 2013, 20, 72-83. [CrossRef]

32. Filter, R.; Farhat, M.; Steglich, M. Tunable graphene antennas for selective enhancement of THz-emission. Opt. Express 2013, 21, 3737-3745. [CrossRef] [PubMed]

33. Zak, A.; Andersson, M.A.; Bauer, M.J.; Matukas, J.; Lisauskas, A.; Roskos, H.G.; Stake, J. Antenna-integrated 0.6 THz FET direct detectors based on CVD graphene. Nano Lett. 2014, 14, 5834-5838. [CrossRef] [PubMed]

34. Xu, Z.; Dong, X.; Bornemann, J. Design of a reconfigurable MIMO system for THz communications based on graphene antennas. IEEE Trans. Terahertz Sci. Technol. 2014, 4, 609-617. [CrossRef]

35. Correas-Serrano, D.J.; Gomez-Diaz, S. Electrically and magnetically biased graphene-based cylindrical waveguides: Analysis and applications as reconfigurable antennas. IEEE Trans. Terahertz Sci. Technol. 2015, 5, 951-960. [CrossRef]

36. Dragoman, M.; Muller, A.A.; Dragoman, D.; Coccetti, F.; Plana, R. Terahertz antenna based on graphene. J. Appl. Phys. 2010, 107, 104313. [CrossRef]

37. Tamagnone, M.; Gómez-Díaz1, J.; Mosig, J.; Perruisseau-Carrier, J. Analysis and design of THz antennas on plasmonic resonant graphene sheets. J. Appl. Phys. 2012, 112, 9151-9154. [CrossRef]

38. Amanatiadis, S.A.; Karamanos, T.D.; Kantartzis, N.V. Radiation efficiency enhancement of graphene THz antennas utilizing metamaterial substrates. IEEE Antennas Wirel. Propag. Lett. 2017, 16, 2054-2057. [CrossRef]

39. Hosseininejad, S.E.; Alarcón, E.; Komjani, N.; Abadal, S.; Lemme, M.C.; Bolívar, P.H.; Cabellos-Aparicio, A. Study of hybrid and pure plasmonic terahertz antennas based on graphene guided-wave structures. Nano Commun. Netw. 2017, 12, 34-42. [CrossRef]

40. Correas-Serrano, D.; Gomez-Diaz, J.S. Graphene-Based Antennas for Terahertz Systems: A review. Forum for Electromagnetic Research Methods and Application Technologies. 2017. Available online: https:/ /arxiv. org/ftp/arxiv / papers/1704/1704.00371.pdf (accessed on 2 May 2018).

41. Abadal, S.; Llatser, I.; Mestres, A.; Lee, H.; Alarcon, E.; Cabellos-Aparicio, A. Time-domain analysis of graphene-based miniaturized antennas for ultra-short-range impulse radio communications. IEEE Trans. Commun. 2015, 63, 1470-1482. [CrossRef]

42. Carrasco, E.; Perruisseau, C.J. Reflectarray antenna at terahertz using graphene. IEEE Antennas Wirel. Propag. Lett. 2013, 12, 253-256. [CrossRef]

43. Chang, Z.; You, B.; Wu, L.; Tang, M.; Zhang, Y. A reconfigurable graphene reflectarray for generation ofvortex THz waves. IEEE Antennas Wirel. Propag. Lett. 2016, 15, 1537-1540. [CrossRef]

44. Shi, Y.; Zhang, Y. Generation of Wideband Tunable Orbital Angular Momentum Vortex Waves Using Graphene Metamaterial Reflectarray. IEEE Access 2017, 6, 5341-5347. [CrossRef]

45. Yu, H.; Yao, Y.; Qu, S. Design of graphene based reflectarray antenna. In Proceedings of the Millimeter Waves and THz Technology Workshop, Caerdydd, UK, 14-15 September 2016; pp. 1-4.

46. You, B.; Zhou, L.; Wu, L. Reconfigurable graphene reflectarray with switchable focus. In Proceedings of the IEEE Mtt-S International Microwave Workshop Series on Advanced Materials and Processes for Rf and Thz Applications, Chengdu, China, 20-22 July 2016; pp. 1-3.

47. Sherrott, M.C.; Pwc, H.; Fountaine, K.T.; Garcia, J.C.; Ponti, S.M.; Brar, V.W.; Sweatlock, L.A.; Atwater, H.A. Experimental demonstration of $230^{\circ}$ phase modulation in gate-tunable graphene-gold reconfigurable mid-infrared metasurfaces. Nano Lett. 2017, 17, 3027-3034. [CrossRef] [PubMed]

48. Zhang, Y.; Tan, Y.W.; Stormer, H.L.; Kim, P. Experimental observation of the quantum hall effect and berry'sphase in graphene. Nature 2005, 438, 201-204. [CrossRef] [PubMed]

49. Deng, L.; Wu, Y.; Zhang, C.; Hong, W.; Peng, B.; Zhu, J.; Li, S. Manipulating of different-polarized reflected waves with graphene-based plasmonic metasurfaces in terahertz regime. Sci. Rep. 2017, 7, 10558. [CrossRef] [PubMed]

50. Falkovsky, L.A. Optical properties of graphene. J. Exp. Theor. Phys. 2008, 115, 012004. [CrossRef]

51. Novoselov, K.S.; Geim, A.K.; Morozov, S.V. Electric field effect in atomically thin carbon films. Science 2004, 306, 666-669. [CrossRef] [PubMed] 
52. Liu, Y.; Wang, P.; Peng, S. Reversibility of diffraction optical path by fresnel theory. J. App. Opt. 2011, 32, 1103-1109.

53. Fan, C.; Choi, W.W.; Yang, W. A Low cross-polarization reflectarray antenna based on SIW slot antenna. IEEE Antennas Wirel. Propag. Lett. 2017, 16, 333-336. [CrossRef]

54. Chaharmir, M.R.; Shaker, J.; Cuhaci, M. Novel photonically-controlled reflectarray antenna. IEEE Trans. Antennas Propag. 2006, 54, 1134-1141. [CrossRef]

55. Carrasco, E.; Tamagnone, M.; Perruisseau, C.J. Tunable graphene reflective cells for THz reflectarrays and generalized law of reflection. Appl. Phys. Lett. 2013, 102, 183-947. [CrossRef]

2018 by the authors. Licensee MDPI, Basel, Switzerland. This article is an open access article distributed under the terms and conditions of the Creative Commons Attribution (CC BY) license (http:/ / creativecommons.org/licenses/by/4.0/). 